\title{
INFLUÊNCIA DO TRATAMENTO TÉRMICO DA ALUMINA NA REAÇÃO DE DECOMPOSIÇÃO DO ISOPROPANOL
}

\author{
M. S. P. MARTINS ${ }^{1}$, J. A. J. RODRIGUES ${ }^{2}$ e G. G. CORTEZ ${ }^{1}$ \\ ${ }^{1}$ Escola de Engenharia de Lorena - USP - Departamento de Engenharia Química \\ ${ }^{2}$ Instituto Nacional de Pesquisas Espaciais-LCP \\ E-mail para contato: cortez@dequi.eel.usp.br
}

\begin{abstract}
RESUMO - A estrutura interna de materiais porosos apresenta grande importância no desenvolvimento de catalisadores eficientes e seletivos. Neste projeto foi obtido um precursor que após tratamento térmico gerou duas aluminas de transição, $\gamma-\mathrm{Al}_{2} \mathrm{O}_{3}$ e $\eta-\mathrm{Al}_{2} \mathrm{O}_{3}$. Estas aluminas foram avaliadas na reação de decomposição do isopropanol. Os resultados mostraram uma alteração na produção de propileno e de éter diisopropílico a partir da evolução da temperatura de calcinação.
\end{abstract}

\section{INTRODUÇÃO}

A alumina é um material estrutural importante, com a ampla aplicação técnica e de enorme importância no campo da catálise heterogênea (Ma e Zhu, 2009; Wang et al., 2009). As aluminas de transição atuam como catalisadores ou como suportes catalíticos para outros metais em inúmeras reações catalíticas. (Gitzen, 1970).

O uso da decomposição de alcoóis é uma prova que os sítios ácido-básico são vastamente estudados (Gervasini e Auroux, 1991).

Este estudo tem como objetivos principais sintetizar um hidróxido de alumínio e calciná-lo a diferentes temperaturas para ser utilizado como catalisador, caracterizar os catalisadores através das técnicas de volumetria de $\mathrm{N}_{2}$ e DRX e avaliar as propriedades ácidas e/ou básicas do catalisador mediante a reação de decomposição do isopropanol.

\section{EXPERIMENTAL}

\subsection{Síntese dos catalisadores}

Sintetizou-se a boemita e a baierita através do método de precipitação com o uso de uma solução de cloreto de alumínio e uma solução de hidróxido de sódio. A solução de $\mathrm{NaOH}$ foi adicionada em um reator batelada sob agitação mecânica de $600 \mathrm{rpm}$ e aquecido a $65{ }^{\circ} \mathrm{C}$. Já a solução de $\mathrm{AlCl}_{3}$ foi adicionada aos poucos, em um período total de $1 \mathrm{~h}$. Realizou-se a lavagem da amostra e depois deixou-a por uma noite na estufa a $50{ }^{\circ} \mathrm{C}$, em seguida peneirouse a massa seca. Esse material foi calcinado até as temperaturas finais de 450, 500, 550 e 600 ${ }^{\circ} \mathrm{C}$. Com esse procedimento, obteve-se os catalisadores identificados por $\mathrm{Al}_{2} \mathrm{O}_{3}$ - $\mathrm{x}$, onde $\mathrm{x}$ representa a temperatura de calcinação. 


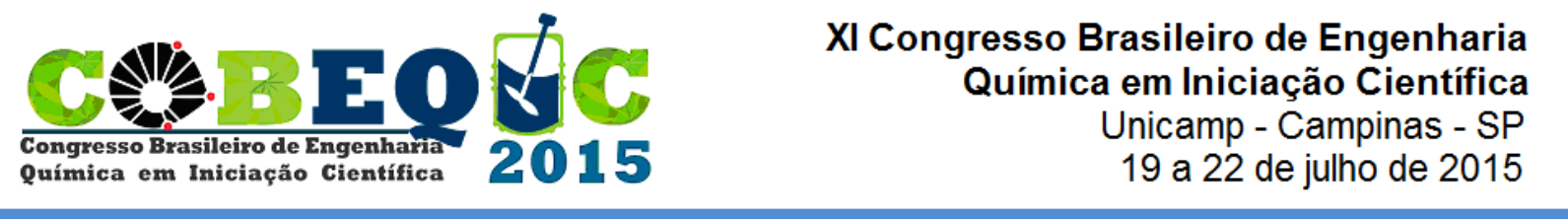

\subsection{Caracterização físico-química}

As medidas de área específica, volume de poros (método BET) e distribuição de volume de poros (método $\mathrm{BJH}$ ) foram determinadas a partir das isotermas de adsorção-dessorção de $\mathrm{N}_{2}$ a $-196{ }^{\circ} \mathrm{C}$. Utilizou-se o equipamento Belsorp II - mini.

As análises por difratometria de raios $\mathrm{X}$ (DRX) foram realizadas empregando-se $\mathrm{o}$ método do pó. Utilizou-se o equipamento da marca Panalytical, modelo Enpyrean. As fases cristalinas foram identificadas com auxílio do programa graph analyzer criado pelo INPE de Cachoeira Paulista.

Para os ensaios de análise termogravimétrica (TGA) e calorimetria exploratória diferencial (DSC) foi utilizada a termobalança da marca TA Instruments, modelo SDT Q600.

As propriedades ácidas e/ou básicas dos catalisadores foram avaliadas através da reação de decomposição do isopropanol. O reator foi alimentado com isopropanol e injetado na tubulação de alimentação de gases afluentes do reator com auxílio de uma bomba (Thermo Separation Products, modelo P100) e diluído em hélio. Os gases efluentes do reator foram analisados por cromatografia gasosa, utilizando-se um cromatógrafo da marca Varian, modelo 3380, equipado com um detector de condutividade térmica (DCT), e conectado em linha com o sistema reacional através de uma válvula de injeção automática. Para separação e análise dos efluentes do reator foi utilizada uma coluna de aço inox empacotada com fase estacionária Poropak-Q $(4,5 \mathrm{~m})$. A taxa específica de formação dos produtos (TEP) foi calculada a partir das equações abaixo:

$$
T E P=\frac{S_{P} \times T R E}{S_{B E T}}
$$

$$
T R E=\frac{X_{A} \times F_{A 0}}{W \times S_{B E T}}
$$

(2)

$$
\begin{aligned}
& X_{A}(\%)=\left(\frac{n_{R}}{n_{R_{0}}}\right) \times 100 \\
& S_{P}(\%)=\left(\frac{n_{R}}{n_{R_{0}}}\right) \times\left(\frac{N_{C P}}{N_{C R}}\right) \times 100,
\end{aligned}
$$

onde TRE é a taxa de reação específica, $\mathrm{X}_{\mathrm{A}}$ é a conversão de isopropanol, $\mathrm{S}_{\mathrm{P}}$ a seletividade dos produtos, $n_{R}$ é o número de mols do isopropanol consumido, $n_{R_{0}}$ é o número de mols de isopropanol na alimentação e ${ }^{N_{C P}}$ e ${ }^{N_{C R}}$ são os números de átomos de carbono presentes nos produtos formados e no isopropanol, respectivamente.

\section{RESULTADOS E DISCUSSÕES}


As áreas específicas $\left(\mathrm{S}_{\mathrm{BET}}\right)$, volume total de poros $(\mathrm{Vp})$ e diâmetro médio dos poros (Dp) dos catalisadores $\mathrm{Al}_{2} \mathrm{O}_{3}$-X são apresentados na Tabela 1. O aumento na temperatura de calcinação da alumina gerou uma diminuição na área específica dos catalisadores. Em contrapartida, com a elevação dessa temperatura, houve um pequeno aumento no volume total de poros e o diâmetro médio dos poros aumentou.

A Figura 1 mostra as isotermas de adsorção-dessorção de $\mathrm{N}_{2}$ a $-196{ }^{\circ} \mathrm{C}$. Para todas as amostras foram observadas isotermas do tipo IV, onde o ramo inferior mostra a quantidade de nitrogênio adsorvido com o aumento da pressão relativa, enquanto que o ramo superior representa a quantidade do gás dessorvido no processo inverso. Esse tipo de isoterma é característico de sólidos mesoporosos e macroporosos, nos quais o processo de evaporação é diferente do processo de condensação (Teixeira et al., 2001).

Tabela 1 - Valores de área específica, volume e diâmetro de poros dos catalisadores.

\begin{tabular}{cccc}
\hline Amostras & $\mathrm{S}_{\mathrm{BET}}\left(\mathrm{m}^{2} / \mathrm{g}\right)$ & $\mathrm{Vp}\left(\mathrm{cm}^{3} / \mathrm{g}\right)$ & $\mathrm{Dp}(\mathrm{nm})$ \\
\hline $\mathrm{Al}_{2} \mathrm{O}_{3}-450{ }^{\circ} \mathrm{C}$ & 263,0 & 0,37 & 5,5 \\
$\mathrm{Al}_{2} \mathrm{O}_{3}-500{ }^{\circ} \mathrm{C}$ & 215,1 & 0,38 & 6,2 \\
$\mathrm{Al}_{2} \mathrm{O}_{3}-550{ }^{\circ} \mathrm{C}$ & 210,8 & 0,39 & 6,6 \\
$\mathrm{Al}_{2} \mathrm{O}_{3}-600{ }^{\circ} \mathrm{C}$ & 185,6 & 0,39 & 8,1 \\
\hline
\end{tabular}

A Figura 2 mostra a distribuição do diâmetro de poros e do volume de poros. Os quatro catalisadores $\mathrm{Al}_{2} \mathrm{O}_{3}$ - $\mathrm{x}$ apresentaram um comportamento monomodal, com distribuição de poros na faixa de 0 a $10 \mathrm{~nm}$, isto é, em sua maioria poros mais estreitos, com alto volume. Isto justifica suas altas áreas superficiais. $\mathrm{O}$ aumento inicial do volume de poros de nitrogênio a baixas pressões relativas é devido à presença de microporos nos catalisadores.

Figura 1- Isotermas de adsorção-dessorção dos suportes $\mathrm{Al}_{2} \mathrm{O}_{3}$-X
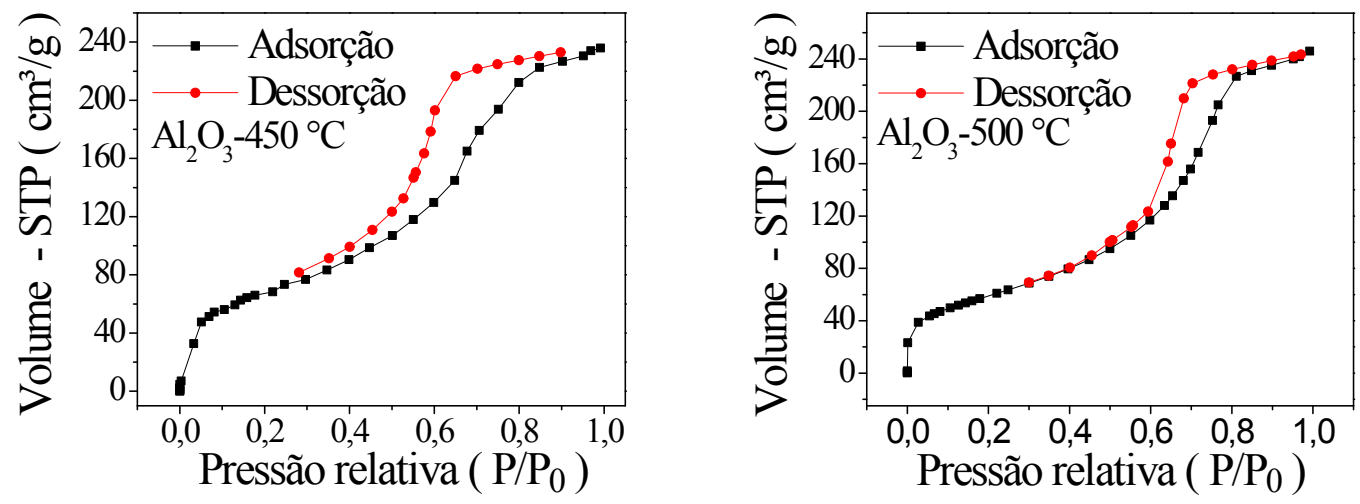

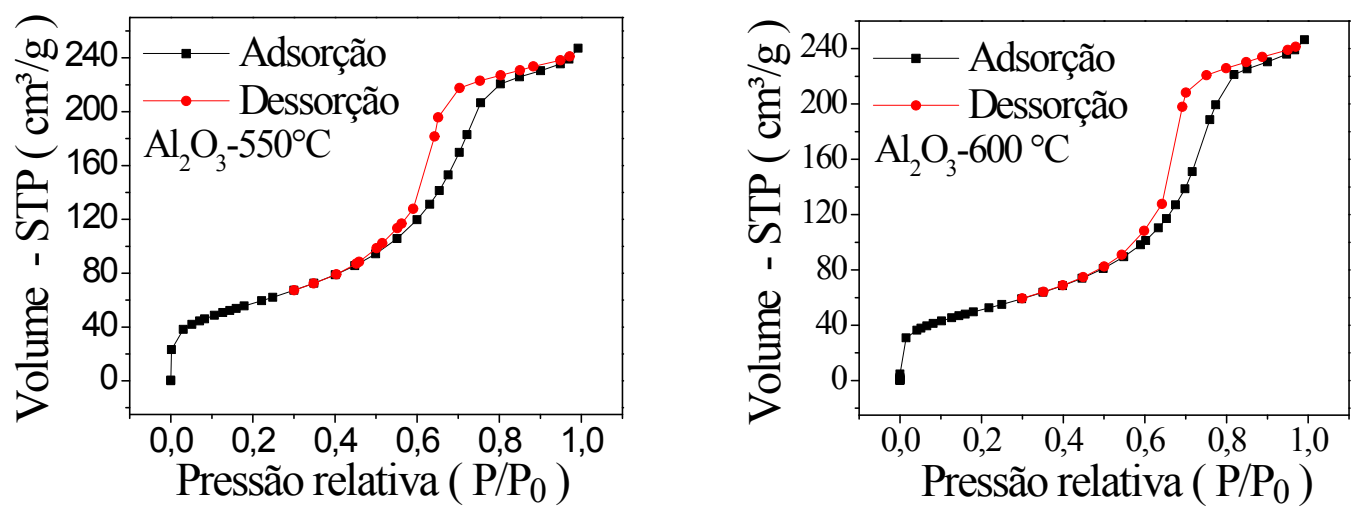

Figura 2 - Volume de poros versus diâmetro de poros para a série $\mathrm{Al}_{2} \mathrm{O}_{3}$-X.
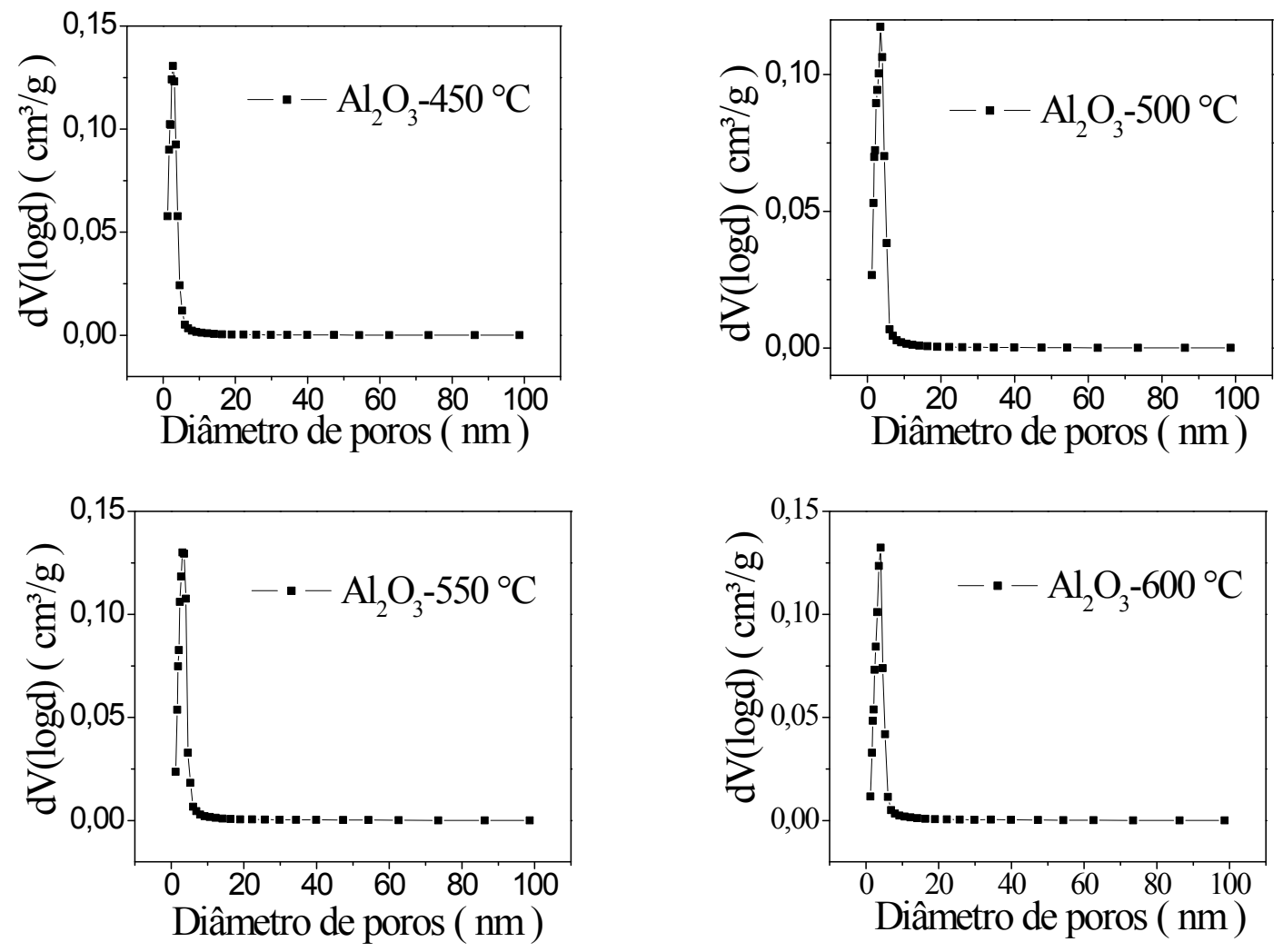

Os picos de difração de raios X (DRX) são apresentados na Figura 3. O precursor seco, preparado a partir do método da precipitação, apresentou as fases boemita (JCPDS, $\mathrm{N}^{\circ}$ 832384 - $\mathrm{AlO}(\mathrm{OH}))$ com estrutura ortorrômbica $(2 \theta=14,492 ; 28,21 ; 38,36 ; 48,94 ; 49,30$; 55,26; 64,14; 64,98 e 72) e baierita (JCPDS, $\mathrm{N}^{\mathrm{o}} 741119$ - $\mathrm{Al}(\mathrm{OH})_{3}$ ) com estrutura monoclínica $(2 \theta=18,81 ; 20,29 ; 27,87 ; 40,65 ; 53,21 ; 57,74 ; 59,35 ; 63,82 ; 64,41$ e 70,76$)$. Assim, como já era esperado, o DRX das amostras calcinadas comprovou a existência das aluminas de transição $\gamma-\mathrm{Al}_{2} \mathrm{O}_{3}$ e $\eta-\mathrm{Al}_{2} \mathrm{O}_{3}$, respectivamente.

A Figura 4 apresenta os resultados da análise termogravimétrica (TGA) e da calorimetria exploratória diferencial (DSC). A TGA permitiu avaliar a variação de massa do 
precursor seco em função da temperatura sob uma atmosfera inerte. Com isso observou-se a perda de cerca de 30\% de massa do precursor seco. Esta diminuição na porcentagem de massa do precursor está relacionada com a evaporação da água. O resultado do DSC é uma curva do fluxo de calor em função da temperatura. A partir desta curva observou-se a presença de três reações endotérmicas, nas temperaturas aproximadas de 100, 280 e $400{ }^{\circ} \mathrm{C}$. Analisando essas temperaturas, concluiu-se que estas reações correspondem, respectivamente, à reação de ebulição da água, à transição da baierita para $\eta-\mathrm{Al}_{2} \mathrm{O}_{3}$ e a transição da boemita para $\eta-\mathrm{Al}_{2} \mathrm{O}_{3}$.

Os resultados da taxa de reação específica (TRE) e da taxa específica de formação dos produtos (TEP), obtidos na reação de decomposição do isopropanol, na temperatura de 240 ${ }^{\circ} \mathrm{C}$, são mostrados na Tabela 2. $\mathrm{O}$ aumento na temperatura de calcinação causou um ligeiro aumento na taxa de reação específica quando a temperatura aumentou de 450 para $500{ }^{\circ} \mathrm{C}$, depois gerou uma diminuição na TRE, tal diminuição está diretamente relacionada à perda de água e a acidez dos produtos formados. Assim, houve primeiramente um pequeno aumento na produção de propeno e de éter diisopropílico, seguido de uma diminuição em suas produções. Sabe-se que a decomposição do isopropanol ocorre através de duas reações paralelas: a desidratação conduzida pelos sítios ácidos, formando propileno e éter diisopropílico e a desidrogenação para formação de acetona sobre sítios básicos ou redox (Heese et al., 1999; Chang, 1999). Assim, de uma maneira geral, os catalisadores $\mathrm{Al}_{2} \mathrm{O}_{3}-\mathrm{x}$ apresentaram predominantemente sítios ácidos, evidenciado pela formação de propeno e éter diisopropílico como produto. A partir da reação de decomposição do isopropanol não é possível estabelecer a natureza dos sítios ácidos de Lewis e Brønsted.

Figura 3 - DRX do precursor seco e das amostras calcinadas. Picos referentes às fases $(\bullet) \gamma-\mathrm{Al}_{2} \mathrm{O}_{3}$,

(口) $\eta-\mathrm{Al}_{2} \mathrm{O}_{3},(\bullet)$ baierita ( $\square$ ) boemita

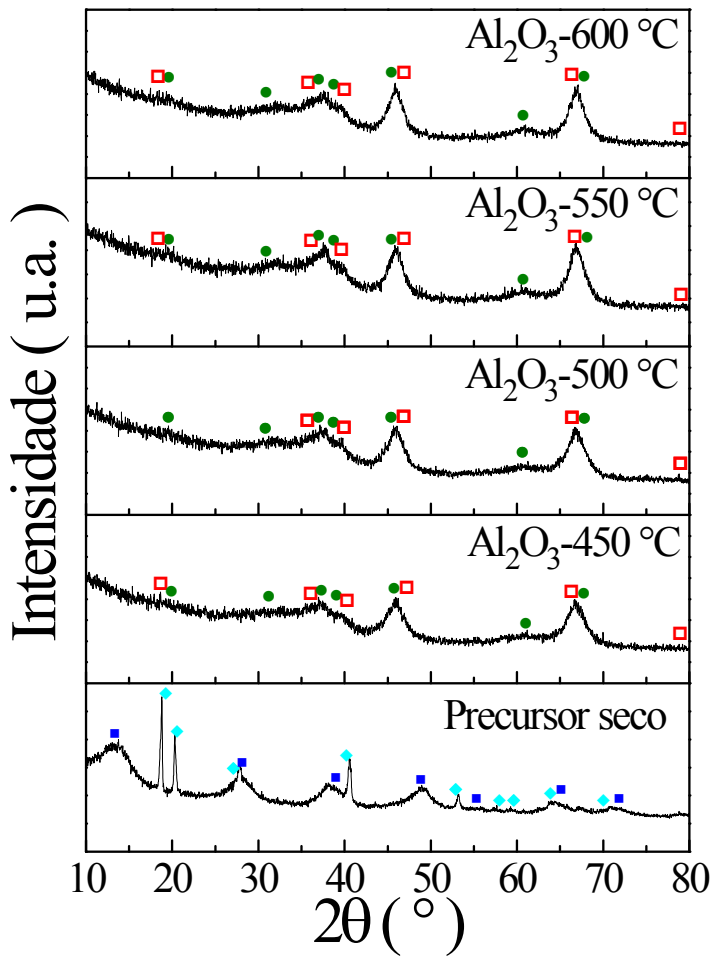

Figura 4 - Resultados da análise termogravimétrica (TGA) e da calorimetria exploratória diferencial (DSC).

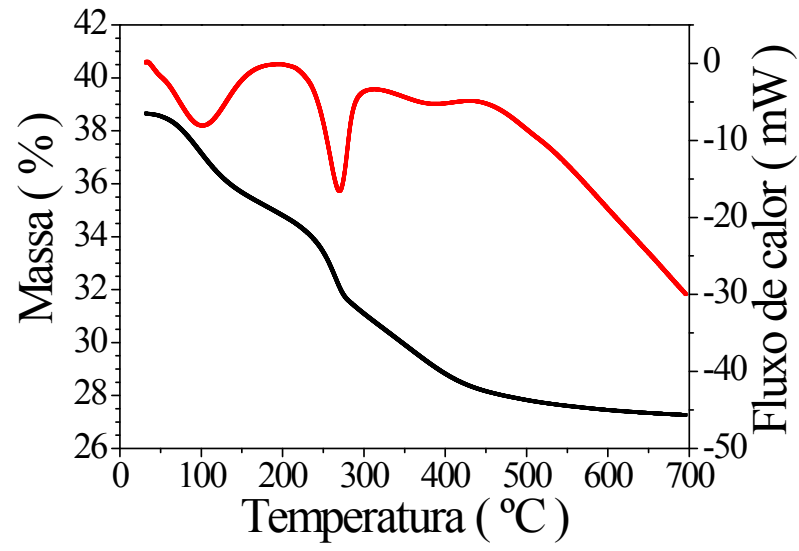


Tabela 2 - Resultados da taxa de reação específica (TER) e da taxa específica de formação dos produtos (TEP) dos catalisadores a $240^{\circ} \mathrm{C}$.

\begin{tabular}{lccc}
\hline \multirow{2}{*}{ Catalisadores } & TER $\left(\mu \mathrm{mol} \cdot \mathrm{m}^{-2} \cdot \mathrm{min}^{-1}\right)$ & \multicolumn{2}{c}{ TEP $\left(\mu \mathrm{mol}^{-2} \cdot \mathrm{min}^{-1}\right) / 240{ }^{\circ} \mathrm{C}$} \\
\cline { 3 - 4 } & & Propeno & Éter diisopropílico \\
\hline $\mathrm{Al}_{2} \mathrm{O}_{3}-450^{\circ} \mathrm{C}$ & 19,0 & 17,0 & 2,0 \\
$\mathrm{Al}_{2} \mathrm{O}_{3}-500^{\circ} \mathrm{C}$ & 21,0 & 18,0 & 3,0 \\
$\mathrm{Al}_{2} \mathrm{O}_{3}-550^{\circ} \mathrm{C}$ & 18,0 & 14,0 & 3,0 \\
$\mathrm{Al}_{2} \mathrm{O}_{3}-600^{\circ} \mathrm{C}$ & 12,0 & 10,0 & 1,0 \\
\hline
\end{tabular}

\section{CONCLUSÕES}

As propriedades texturais dos catalisadores A12O3-x foram modificadas pelo aumento da temperatura de calcinação. Assim, em cada temperatura de calcinação, os catalisadores estudados apresentaram características diferentes de área específica, de volume de poros e de diâmetro de poros. A síntese do precursor revelou, no DRX, um material composto por boehmita e baierita. Todos os catalisadores calcinados apresentaram em DRX aluminas de transição tipo $\gamma$-A12O3 e $\eta-\mathrm{A} 12 \mathrm{O} 3$. No resultado da análise termogravimétrica (TGA) e da calorimetria exploratória diferencial (DSC), observou-se uma grande perda de massa e a presença de três reações endotérmicas, devido à eliminação da água da estrutura do óxido de alumínio. A partir da reação de decomposição do isopropanol, conclui-se que os catalisadores apresentaram basicamente sítios ácidos, evidenciado pela formação de propeno e éter diisopropílico. Além disso, verificou-se que o aumento na temperatura de calcinação diminuiu a atividade catalítica pela diminuição de sítios ácidos superficiais.

\section{AGRADECIMENTOS}

Ao LCP-INPE de Cachoeira Paulista/SP e à FAPESP (2013/23400-4).

\section{REFERÊNCIAS}

CHANG, T. Controversy over MTBE in gasoline rages on. Oil Gas J., v. 97, p. 34-36, 1999.

GERVASINI, A.; AUROX, A., Acidity and basicity of metal oxide surfaces II. Determination by catalytic decomposition of isopropanol. J. Catal., v. 131, p. 190-198, 1991.

GITZEN, W. H., Alumina as a Ceramic Material. J. Am. Ceram. Soc., p. 121-199, 1970.

HEESE, F. P.; DRY, M.; MOLLER, K. P. Single stage synthesis of diisopropyl ether - An alternative octane enhancer for lead-free petrol. Catal. Today, v. 49, p. 327-335, 1999.

MA, M.G.; ZHU, J.F., A facile solvothermal route to synthesis of $\gamma$-alumina with bundle-like and flower-like morphologies, Mater. Lett., v. 63, p. 881-883, 2009.

TEIXEIRA, V. G.; COUTINHO, F. M. B.; GOMES, A. S. Principais métodos de caracterização da porosidade de resinas à base de divinilbenzeno. Quim. Nova, v. 24(6), p. $808-818,2001$. 
WANG, Y.; WANG, J.; SHEN, M.; WANG, W., Synthesis and properties of thermostable $\gamma$ alumina prepared by hydrolysis of phosphide aluminum. J. Alloys Compd., v.467, p.405-412, 2009. 